\title{
ANOMALOUS SYNCHRONIZATION OF SPATIALLY EXTENDED CHAOTIC SYSTEMS IN THE PRESENCE OF ASYMMETRIC COUPLING
}

\author{
S. BOCCALETTI and C. MENDOZA \\ Instituto Nazionale di Ottica Applicata, Largo E. Fermi, 6 I50125 Florence, Italy. \\ J. BRAGARD \\ Dept. of Physics and Applied Math., Universidad de Navarra, E31080 Pamplona, Spain
}

\author{
Received (11 January 2005) \\ Revised (24 February 2005) \\ Accepted (16 March 2005)
}

Communicated by Werner Ebeling and Bernardo Spagnolo

\begin{abstract}
This paper describes the effects of an asymmetric coupling in the synchronization of two spatially extended systems. Namely, we report the consequences induced by the presence of asymmetries in the coupling configuration of a pair of one-dimensional fields obeying Complex Ginzburg Landau equations. While synchronization always occurs for large enough coupling strengths, asymmetries have the effect of enhancing synchronization and play a crucial role in setting the threshold for the appearance of the synchronized dynamics, as well as in selecting the statistical and dynamical properties of the synchronized motion. We discuss the process of synchronization in the presence of asymmetries by using some analytic expansions valid for a regime of soft spatial temporal chaos (i.e. phase turbulence regime). The influence of phase singularities that break the validity of the analysis is also discussed.
\end{abstract}

Keywords: Synchronization; extended systems.

\section{Introduction}

In recent years, synchronization of complex systems have generated a great echo in the scientific community [1], as well as in the literature oriented to lay audiences [2]. A relevant and counterintuitive result is that an increase in coupling strength between two complex systems does not necessarily induce a better degree of synchronization. An example is the anomalous behavior observed in the frequency synchronization between a limit-cycle and a time chaotic oscillator [3,4], where increasing the coupling leads initially to an increase of the frequency difference.

In this paper we will analyze anomalous frequency synchronization (AFS) oc- 
curring for space extended system obeying Complex Ginzburg Landau Equation (CGLE) in Phase Turbulence (PT). PT is a regime where the chaotic behavior of the complex field is dominated by the dynamics of its phase, while the dynamics of the modulus is slaved to the phase variations. The modulus is changing only smoothly throughout the system extension and is always bounded away from zero. We will show that depending on the value of the coupling asymmetry the system of two coupled CGLE exhibits anomalous or monotonic synchronization. The monotonic synchronization means that the frequency difference is decreasing monotonously to zero as the coupling is increased. In the following, we will show that the presence of phase defects in spatially extended chaotic oscillators has the role of enhancing the anomaly in frequency synchronization with respect to the case of merely time chaotic oscillators. A detailed account of the different dynamics of the CGLE as well as an introduction to synchronization phenomena can be found in Ref. $[6,7]$. In order to be able to use analytical tools, we will study the case of phase turbulence which is more amenable to analytical approaches. For this purpose, we set the parameters in Eq.(1) to be $\alpha=2, \beta_{1}=-0.75$ and $\beta_{2}=-0.9$. This will permit to perform detailed analytical study of the synchronization process and in particular allow us to predict a priori which values of the asymmetry will lead to regular (FS) or AFS.

Let us recall the model equation under study:

$$
\begin{aligned}
\dot{A}_{1,2}= & A_{1,2}+(1+i \alpha) \partial_{x x} A_{1,2}-\left(1+i \beta_{1,2}\right)\left|A_{1,2}\right|^{2} A_{1,2} \\
& +\frac{c}{2}(1 \mp \theta)\left(A_{2,1}-A_{1,2}\right) .
\end{aligned}
$$

Here, $A_{1,2}(x, t)=\rho_{1,2}(x, t) e^{i \phi_{1,2}(x, t)}$ are two complex field of modulus $\rho_{1,2}(x, t)$ and phases $\phi_{1,2}(x, t)$, dots denote temporal derivatives, $\partial_{x x}$ stays for the second derivative with respect to the space variable $0 \leq x \leq L, L$ is the system extension, $\alpha$ and $\beta_{1,2}$ are real parameters that measure linear and nonlinear dispersion [8], $c$ represents the coupling strength and $\theta$ is a parameter accounting for asymmetries in the coupling.

The numerical simulations presented in this article have been done using a standard Cranck-Nicholson Adams-Bashforth scheme [9] with a time step $\delta t=10^{-2}$ and grid size $\delta x=0.25$. The spatial extension of the system is $L=100$ and periodic boundary conditions are imposed.

Let us now define the natural average frequencies of the two CGLE

$$
\Omega_{1,2}=\lim _{t \rightarrow \infty} \frac{<\phi_{1,2}(x, t)>_{x}}{t}
$$

where $\phi$ is defined in $\Re$ rather than in $[0,2 \pi]$ and $\left(<>_{x}\right)$ denotes a spatial average. The sub-indices 1,2 refer to $A_{1}$ and $A_{2}$, respectively. Figure 1 reports the variation of the natural frequency of a single CGLE as a function of the parameter $\beta$. The region where the CGLE is in the PT regime is for $\beta>-0.97$. From Eq. (2), one can define the frequency difference $\Delta \Omega \equiv \Omega_{2}-\Omega_{1}$ which is the indicator for characterizing frequency synchronization.

Figure 2 reports the frequency difference $(\Delta \Omega)$ as a function of the coupling parameters $(c, \theta)$ and indicates that the transition to a frequency locked state $(\Delta \Omega=$ $0)$ can occur in a regular $(\Delta \Omega$ is a monotonically decreasing function of $c$ ) or in an 


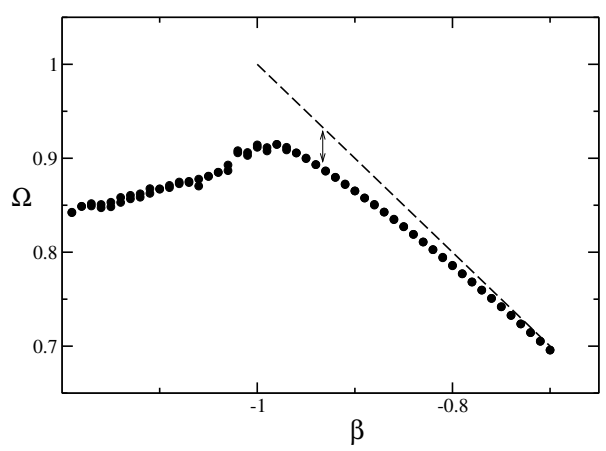

Fig 1. Natural average frequency $\Omega$ vs. $\beta$ for $\alpha=2$. The filled dots report the values obtained from simulations of the CGLE. The dashed line $\Omega=-\beta$ indicates the prediction given by the dispersion relation of the plane wave solutions of the CGLE with zero wavenumber $A(x, t)=e^{i \Omega t}$.

anomalous fashion $(\Delta \Omega$ increases initially with $c)$, depending upon the level of asymmetry in the coupling configuration. The critical point at $\theta_{c r}=-0.09$ draws the separation between regular $\left(\theta_{c r}<\theta \leq 1\right)$ and anomalous $\left(-1 \leq \theta<\theta_{c r}\right)$ phase synchronization and is represented by an arrow in Fig. 2. This value of the asymmetry marks the separation between the two regimes i.e. regular and anomalous and will be determined analytically in the next section.

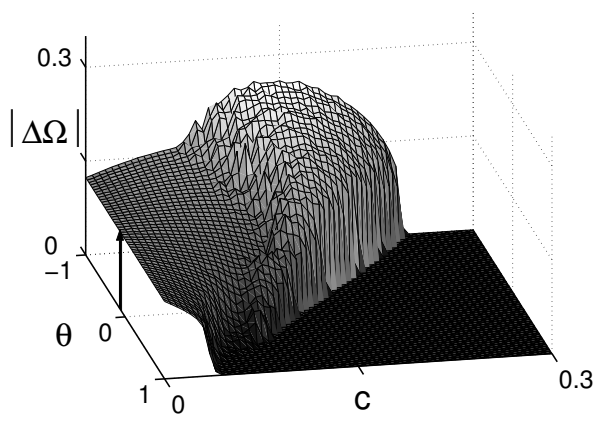

Fig 2. Frequency mismatch $|\Delta \Omega| v s$. the parameter space $(c, \theta)$ for Eq. (1). The parameters are $\alpha=2 ; \beta_{1}=-0.75$ and $\beta_{2}=-0.9$. Each value of $\Delta \Omega$ is an average over a time $\mathrm{t}=15000$ after a large transient has elapsed $\mathrm{T}=6.000$.

\section{Anomalous Frequency Synchronization}

In the PT regime the dynamics of the CGLE can be described by a phase equation [10]. The equation for the phase dynamics of the single CGLE has been derived by 
Sakaguchi [11] and reads as:

$$
\begin{aligned}
\dot{\phi}= & t_{1} \phi_{x x}+t_{2} \phi_{x}^{2}+t_{3} \phi_{x x x x} \\
& +t_{4} \phi_{x} \phi_{x x x}+t_{5} \phi_{x x}^{2}+t_{6} \phi_{x}^{2} \phi_{x x}
\end{aligned}
$$

where $t_{1}=1+\alpha \beta, t_{2}=\beta-\alpha, t_{3}=-\alpha^{2}\left(1+\beta^{2}\right) / 2, t_{4}=-2 \alpha\left(1+\beta^{2}\right), t_{5}=-\alpha\left(1+\beta^{2}\right)$ and $t_{6}=-2\left(1+\beta^{2}\right)$. Equation (3) is obtained by doing a systematic asymptotic expansion of Eq. (1) with $c=0$, in powers of $\phi_{x}$.

As we are interested in a maximum reduction of the dynamics of the two coupled CGLE, we will approximate the effect of the spatial terms in Eq. (3) by an adequate additive noise in the equation of two coupled oscillators Eq. (4)

$$
\begin{aligned}
& \dot{\phi}_{1}=-\beta_{1}-c_{1}\left[\beta_{1}(\cos \varphi-1)-\sin \varphi\right]+\eta_{1}, \\
& \dot{\phi}_{2}=-\beta_{2}-c_{2}\left[\beta_{2}(\cos \varphi-1)+\sin \varphi\right]+\eta_{2},
\end{aligned}
$$

where $\varphi=\phi_{2}-\phi_{1}$ represents the phase difference between the two oscillators, $c_{1}=c(1-\theta) / 2, c_{2}=c(1+\theta) / 2$, and $\eta_{1}, \eta_{2}$ are additive noise.

The drastic reduction from the partial differential equations (1) to the two stochastic differential equations (4) will only be justified if we can extract some useful information from Eq. (4). In particular, we are interested in reproducing the regular and anomalous frequency synchronization that has been observed in the full coupled CGLE Eq. (1).

If we are interested in a first order approximation theory, we can keep only the first three terms in the right-hand-side of Eq.(3). The spatial average of the phase variation

$$
<\dot{\phi}>_{x}=t_{2}<\phi_{x}^{2}>_{x} .
$$

takes a very simple form due to the spatial periodic boundary conditions. The simulations of Eq. (3) show that the phase $\phi$ is drifting linearly with time $(<$ $\phi>{ }_{x} \approx s t+b$, where $s$ is the slope of the linear drift evaluated by performing a linear fit. The frequency estimate from the phase approximation model is therefore given by $\Omega=-\beta+s$. In Fig. 1 the small correction $s$ given by Eq. (3) has been represented by a double arrow between the line $\Omega=-\beta$ and the results of the simulations of the full CGLE in the PT regime $(-0.9<\beta<-0.7)$.

The term $<\phi_{x}^{2}>$ (from now on referred to as T2) is responsible for the deviation of the frequency between the linear dispersion relation and the frequency given by the full CGLE Eq. (1).

The time evolution of T2 is chaotic and it is displayed in Fig. 3. From the time evolution, we can extract a probability distribution function (PDF) that can be conveniently fitted by a Gaussian (as shown in Fig. 4(a)). Furthermore, the time correlation function for T2 is also calculated and reported in Fig. 4(b). The PDF is well approximated by a Gaussian distribution. (see Fig. 4). The next step is to use the fluctuation characteristics of the term T2 and use it as an additive colored Gaussian noise in Eq. (4). The statistical properties of this noise is obviously dependent of the parameters $\alpha$ and $\beta$ of the original CGLE. We have calculated from extensive numerical simulations (on nine different realizations) that for $\beta_{1}=-0.75$ $\left(\beta_{2}=-0.9\right)$ we have that the mean of the PDF is $\mu_{1}=2.9210^{-3}\left(\mu_{2}=1.1410^{-2}\right)$, 


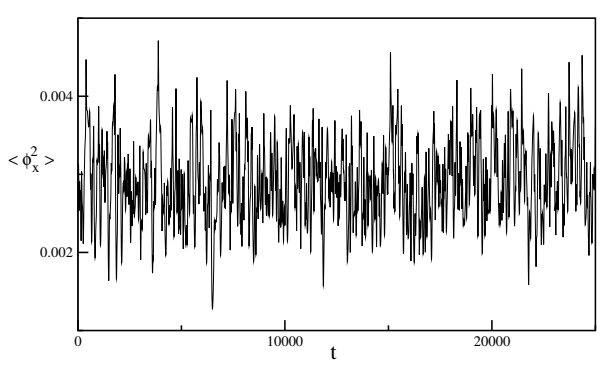

Fig 3. Time evolution of $\left\langle\phi_{x}(x, t)^{2}>_{x}\right.$ from Eq. (1) with $c=0, \alpha=2, \beta=-0.75$.

the standard deviation is $\sigma_{1}=5.4610^{-4}\left(\sigma_{2}=1.710^{-3}\right)$ and the correlation time is $\left.\tau_{1}=51.2\left(\tau_{2}=15.3\right)\right]$. Note that, as $\beta$ is increased (in absolute value), the system becomes more and more chaotic, thus the correction to the frequency is larger (as indicated by a larger $\mu$ ), the fluctuations are higher (as indicated by a larger $\sigma$ ) and the correlation time of the signal decays (as confirmed by a lower value of $\tau$ ).
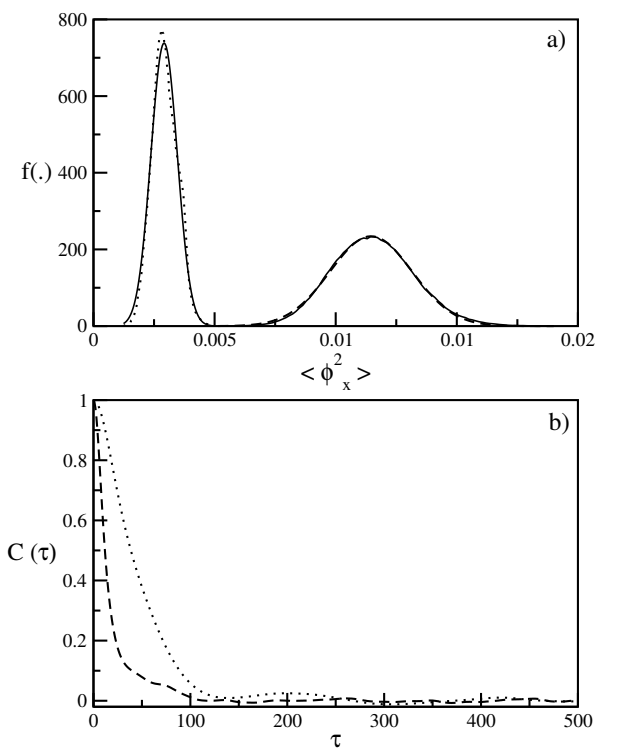

Fig 4. Probability distribution function of the term T2 (a), and its time correlation function (b), calculated from Eq. (1) with $c=0, \alpha=2$, and $\beta=-0.75$ (dotted lines) and $\beta=-0.9$ (dashed lines). In (a), we also draw with solid lines the two Gaussian PDFs that provide the best fit for the two distributions.

The characterization of the noise permits the drastic reduction from the two 
coupled CGLE Eq. (1) to two single differential equations (Stuart-Landau type) plus some additive noise Eq. (4).

Let us now demonstrate that we get some understanding of mechanisms behind AFS from these reduced equations (4).

Analytical study of Eqs. (4) can be performed, following what done in Ref. $[3,4,12]$. Namely, by neglecting the noise terms and subtracting Eqs. (4), one is able to write an equation for $\dot{\varphi}$ in a closed form. Then frequency synchronization is:

$$
|\Delta \Omega|=\sqrt{B_{-}^{2}+c B_{-}\left[\theta B_{+}-B_{-}\right]+c^{2}},
$$

where $B_{+} ;\left(B_{-}\right)$stays for $\beta_{1}+\beta_{2} ;\left(\beta_{1}-\beta_{2}\right)$, respectively. The interest of expression (6) is that one can analytically estimate the transition point between FS and AFS. Indeed, the slope of the detuning at zero coupling indicates regular (if negative) or anomalous (if positive) frequency synchronization. It is straightforward to calculate the value of $\theta$ for the transition between FS and AFS:

$$
\theta_{c r}=\frac{B_{-}}{B_{+}}=\frac{\beta_{1}-\beta_{2}}{\beta_{1}+\beta_{2}}
$$

For the particular case treated here $\left(\beta_{1}=-0.75\right.$ and $\left.\beta_{2}=-0.9\right)$, we have $\theta_{c r}=-1 / 11 \approx-0.09$, in perfect agreement with what found numerically for the full CGLE model and reported in Fig. 2. That means that for $\theta_{c r}<\theta \leq 1$, we have regular FS. Conversely, in the range $-1 \leq \theta<\theta_{c r}$, we have AFS.
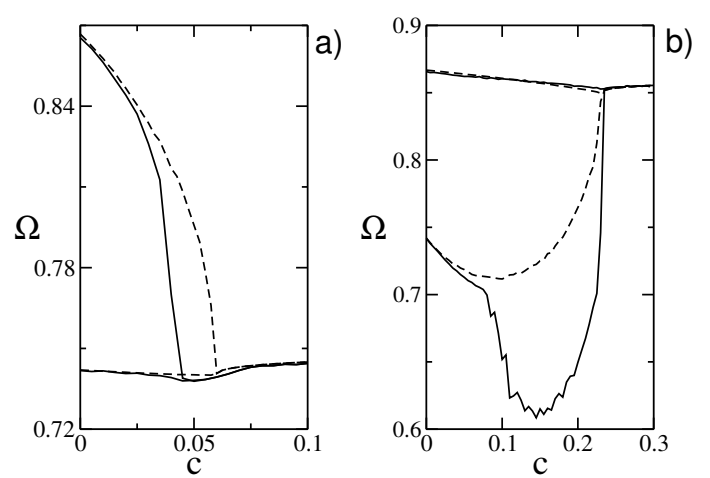

Fig 5. Mean frequencies $\Omega_{1,2}$ calculated for the full CGLE (1) (solid line) and the effective SDE (4) model (dashed line). (a) $\theta=0.88$ (regular FS). (b) $\theta=-0.88$ (AFS) $\left(\beta_{1}=-0.75, \beta_{2}=-0.9\right)$.

Finally, we compare the numerical integration of the SDE (4) and of the full CGLE (1). In Fig. 5 we report the frequencies $\Omega_{1}, \Omega_{2} v s$. the coupling strength $c$ for two asymmetric coupling configurations. Namely, Fig. 5(a) [Fig. 5(b)] refers to the case of regular FS at $\theta=0.88$ (of AFS at $\theta=-0.88$ ). The agreement between the SDE (4) and the full CGLE (1) is very good at low coupling strengths. However, for larger values of $c$, we observe an increasing difference between the two cases, which is especially pronounced in the case of AFS. In particular, the full CGLE shows an enhancement of the anomaly with respect to the SDE Eq. (4). 
a)

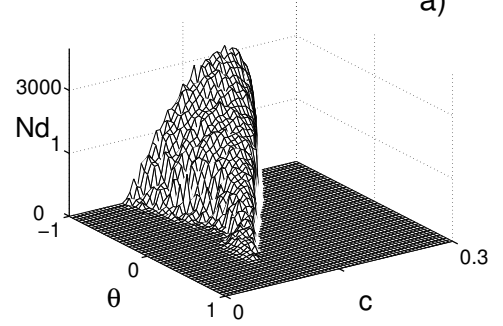

b)

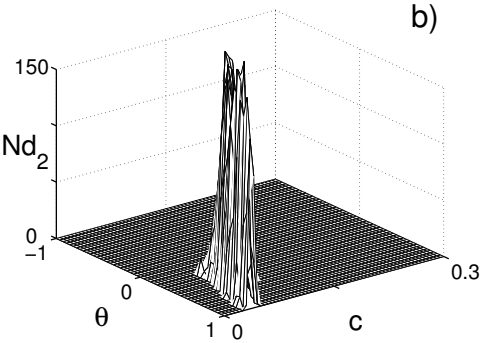

Fig 6. Total number of defects appearing in the coupled CGLE systems (a) corresponds to the system 1, (b) corresponds to the system 2 .

In order to identify the reasons for such a difference, we point out that the derivation of the reduced SDE model is rigorous only in the absence of coupling. While one can reasonably expect that the validity of such reduction would hold also for small values of $c$, at larger coupling strengths intrinsic spatial effects become dominant in the dynamics of the coupled fields. In particular, even though initially the two fields are set in PT regimes, an intermediate coupling induces the presence of a finite number of phase defects (points where locally and instantaneously the amplitude $\rho(x, t)$ vanishes), as it appears from Fig. 6. Phase defects are objects inherent to the spatially extended nature of the system and cannot be retrieved in a SDE model. These objects are therefore leading to a violation of one of the basic assumption of our reduction i.e. the absence of phase defects. Even if the reduction is no longer valid in the presence of phase defects, one can use an ad hoc rescaling to compare the frequenor mivan hr the rodurod madol and the full equation.

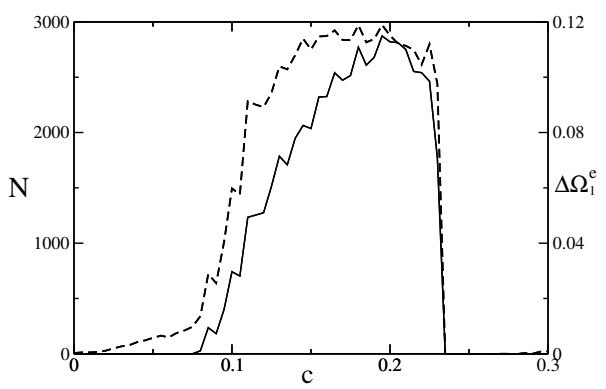

Fig 7. Number of defects in system 1 (solid line-left ordinate) and frequency mismatch $\Delta \Omega_{1}^{e}$ (dashed line-right ordinate, see text for definition) vs. $c$ for $\theta=-0.88$ (AFS).

Phase defects are entirely responsible for the frequency mismatch observed in Fig. 5. Calling $\Omega_{1}^{e f f}\left(\Omega_{1}\right)$ the mean frequency of system 1 as calculated with reference to the SDE model (the full CGLE model), in Fig. 7 we show that for $\theta=$ -0.88 (AFS), the 1:1 correlation between the frequency mismatch $\Delta \Omega_{1}^{e}=\Omega_{1}^{\text {eff }}-\Omega_{1}$ and the number of defects appearing in system 1 is indeed remarkable, indicating that a simple correction of the frequency proportional to the defect numbers is 
enough to produce an excellent agreement between the SDE and the full CGLE models for the whole range of $c$.

\section{Conclusions}

To summarize, the comparison between two time dependent oscillators and spatially extended oscillators cannot be conducted without taking high care of phase defects that may appear in the process of coupling two equations that were initially (for $c=0)$ free of phase defects. These last objects are inherent to space extended system and must be taken into account if we want to study synchronization if they are present in the system. In particular, AFS experiences a further enhancement by phase defects, while in the case of regular FS, the presence of defects shifts down the threshold for synchronization, allowing for an easier frequency locking.

\section{Acknowledgments}

Work partly supported by EU Contract n. HPRN-CT-2000-00158 (COSYC of SENS), MIUR-FIRB project n. RBNE01CW3M-001 and MCYT project (Spain) n. BFM2002-02011 (INEFLUID).

\section{References}

[1] For a comprehensive review on the subject see: S. Boccaletti, J. Kurths, G. Osipov, D. Valladares and C. Zhou, Phys. Rep. 366 (2002), 1, and references therein.

[2] S. Strogatz, Sync: The Emerging Science of Spontaneous Order, Hyperion Press (2003).

[3] E. Montbrio and B. Blasius, Chaos 13 (2003), 291.

[4] B. Blasius, E. Montbrió and J. Kurths, Phys. Rev. E 67 (2003), 035204R.

[5] M. Cross and P. Hohenberg, Rev. Mod. Phys. 65 (1993), 851.

[6] J. Bragard, S. Boccaletti and H. Mancini, Phys. Rev. Lett. 91(6), 064103 (2003).

[7] J. Bragard, S. Boccaletti, C. Mendoza, H. G. E. Hentschel and H. Mancini, Phys. Rev. E 70 (2004), 036219.

[8] I. Aranson and L. Kramer, Rev. Mod. Phys. 74 (2002), 99-143.

[9] W. H. Press, S. A. Teukolsky, W. T. Vetterling, B. P. Flannery, The Art of Scientific Computing, Cambridge Univ. Press (1992).

[10] Y. Kuramoto and T. Tsuzuki, Prog. Theor. Phys. 55 (1976), 356.

[11] H. Sakaguchi, Prog. Theor. Phys. 84 (1990), 792.

[12] C. Mendoza, Ph.D. Thesis, University of Navarre (2003).

[13] E. Helfand, Bell Sys. Tech. J. 58 (1979); H. Greenside and E. Helfand, Bell Sys. Tech. J. 60 (1981).

[14] J. García-Ojalvo, G. M. Sancho and L. Ramírez-Piscina, Phys. Rev. A 46 (1992), 4670.

[15] D. G. Aranson, G. B. Ermentrout and N. Kopell, Physica D 41 (1990), 403. 